\title{
A Statewide Survey of PPO-Inhibitor Resistance and the Prevalent Target-Site Mechanisms in Palmer amaranth (Amaranthus palmeri) Accessions from Arkansas
}

\author{
Vijay K. Varanasi†, Chad Brabham†, Jason K. Norsworthy, Haozhen Nie, Bryan G. Young, \\ Michael Houston, Tom Barber, and Robert C. Scott*
}

Palmer amaranth is one of the most problematic weeds in the midsouthern United States, and the evolution of resistance to protoporphyrinogen oxidase (PPO) inhibitors in biotypes already resistant to glyphosate and acetolactate synthase (ALS) inhibitors is a major cause of concern to soybean and cotton growers in these states. A late-season weed-escape survey was conducted in the major row crop-producing counties (29 counties) to determine the severity of PPO-inhibitor resistance in Arkansas. A total of 227 Palmer amaranth accessions were sprayed with fomesafen at $395 \mathrm{~g} \mathrm{ha}^{-1}$ to identify putative resistant plants. A TaqMan qPCR assay was used to confirm the presence of the $\Delta \mathrm{G} 210$ codon deletion or the R128G/M (homologous to R98 mutation in common ragweed) target-site resistance mechanisms in the PPX2 gene. Out of the 227 accessions screened, 44 were completely controlled with fomesafen, and 16 had only one or two severely injured plants $(\geq 98 \%$ mortality) when compared with the 1986 susceptible check (100\% mortality). The remaining 167 accessions were genotypically screened, and $82(49 \%)$ accessions were found to harbor the $\Delta \mathrm{G} 210$ deletion in the PPX2 gene. The R128G was observed in $47(28 \%)$ out of the 167 accessions screened. The mutation R128M, on the other hand was rare, found in only three accessions. About $13 \%$ of the accessions were segregating for both the $\Delta \mathrm{G} 210$ and R128G mutations. Sixteen percent of the tested accessions had mortality ratings $<90 \%$ and did not test positive for the $\Delta \mathrm{G} 210$ or the R128G/M resistance mechanisms, indicating that a novel target or non-target site resistance mechanism is likely. Overall, PPO inhibitor-resistant Palmer amaranth is widespread in Arkansas, and the $\Delta \mathrm{G} 210$ resistance mechanism is especially dominant in the northeast corridor, while the R128G mutation is more prevalent in counties near Memphis, TN.

Nomenclature: Fomesafen; Palmer amaranth, Amaranthus palmeri S. Wats.; cotton, Gossypium hirsutum L.; soybean, Glycine $\max (\mathrm{L}$.$) Merr.$

Key words: Molecular assay, PPO inhibitors, whole-plant screen.

Palmer amaranth is ranked as the most troublesome and one of the most common weeds found in North America (Wychen 2016). Palmer amaranth is especially prevalent in the midsouthern United States, and its control is a major concern of soybean and cotton growers and consultants in these states (Prostko 2011; Riar et al. 2013). Control of Palmer amaranth has become increasingly difficult over the past decade because of the widespread distribution

\footnotetext{
DOI: $10.1017 /$ wsc. 2017.68

* First, second, third, and sixth authors: Postdoctoral Research Associate, Postdoctoral Research Associate, Professor, and Graduate Student, Department of Crop, Soil, and Environmental Sciences, University of Arkansas, Fayetteville, AR 72704; fourth and fifth authors: Postdoctoral Research Assistant and Professor, Department of Botany and Plant Pathology, Purdue University, West Lafayette, IN 47907; seventh and eighth authors: Professor and Professor, Department of Crop, Soil, and Environmental Sciences, University of Arkansas Lonoke Agricultural Center, Lonoke, AR 72086. Corresponding author's E-mail: varanasi@uark.edu

$\dagger$ These authors made equal contributions.
}

of biotypes resistant to acetolactate synthase (ALS) inhibitors and glyphosate in the South (Bond et al. 2006; Heap 2017). As a consequence, growers have become increasingly dependent on PRE and POST applications of protoporphyrinogen oxidase (PPO) inhibitors, and this appears to be a commonly adopted weed management tactic for control of ALS inhibitor-resistant and glyphosate-resistant broadleaf weeds in soybean and cotton (Owen and Zelaya 2005; Rousonelos et al. 2012).

PPO-inhibiting herbicides prevent the conversion of protoporphyrinogen IX to protoporphyrin IX by the plastid-localized PPO enzyme (PPX2) (Matringe et al. 1989; Jacobs and Jacobs 1993). PPO inhibitorresistant Palmer amaranth was first discovered in 2011 in Arkansas and then in Tennessee and Illinois in 2015 and 2016, respectively (Heap 2017). Initially, the mechanism of resistance to PPO inhibitors in Palmer amaranth was documented as being the same as in common waterhemp (Amaranthus rudis Sauer) (Salas et al. 2016; Thinglum et al. 2011). The target-site

Varanasi et al.: Prevalence of PPO resistance • 149 
resistance mechanism involves a codon deletion in the PPX2 gene, resulting in the loss of a glycine residue at the $210^{\text {th }}$ position $(\Delta \mathrm{G} 210)$ of the PPO enzyme (Lee et al. 2008; Patzoldt et al. 2006). Giacomini et al. (2017) reported two new mutations encoding for a glycine $(\mathrm{G})$ or a methionine $(\mathrm{M})$ instead of an arginine at the $128^{\text {th }}(\mathrm{R} 128)$ amino acid residue (referred to as R98 in their study) in the PPO enzyme of Palmer amaranth. The R128 amino acid is homologous to the R98 in common ragweed (Ambrosia artemisiifolia L.), in which an R98 to leucine amino acid change was shown to confer resistance to fomesafen (Rousonelos et al. 2012; Salas et al. 2017). The difference in the amino acid number is due to the signal peptide (30 aa in length) found in the PPX2 protein of Amaranthus spp. but not in common ragweed (Dayan et al. 2010; Rousonelos et al. 2012).

The R128G/M mutations in Palmer amaranth were identified in an accession from Woodruff County in Arkansas and in two accessions from Lauderdale and Shelby counties in Tennessee (Giacomini et al. 2017). Interestingly, the Woodruff County accession was segregating for the $\Delta \mathrm{G} 210$ deletion and the R128G mutation, and this accession was shown to exhibit cross-resistance to the PPO-inhibiting herbicides fomesafen (diphenylethers), flumioxazin ( $N$-phenylphthalimide), oxadizon (oxadiazole), sulfentrazone (triazolinone), and saflufenacil (pyrimidindione), and presumably to other herbicides from their respective chemical families (Schwartz-Lazaro et al. 2017).

A 2016 survey of soybean consultants from Arkansas, Louisiana, southeast Missouri, Mississippi, and Tennessee found $79 \%$ of the hectares received multiple applications of PPO inhibitors in the past $3 \mathrm{yr}$, and $69 \%$ of the consultants suspected PPO inhibitor-resistant Palmer amaranth in at least one of their scouted fields (JK Norsworthy, personal observations). Thus, a large-scale screen of 227 Palmer amaranth accessions collected from the major row crop-producing counties (29 counties) in Arkansas was conducted to determine the severity of PPO-inhibitor resistance in Arkansas and to further identify the dominant resistance mechanism(s) in these accessions.

\section{Materials and Methods}

Plant Material. A late-season weed-escape survey was conducted in the fall of 2016. Inflorescences from Palmer amaranth accessions were collected from crop production fields (mainly soybean) across the state of Arkansas by our research group, growers, consultants, or extension agents and sent to the Altheimer Laboratory at the University of Arkansas, Fayetteville, AR.
The GPS coordinates for all accessions were recorded. In total, 227 accessions were screened for PPOinhibitor resistance, and the seed lot for each accession, on average, was created by threshing 10 inflorescences.

PPO-Inhibitor Resistance Screen. In the greenhouse, each accession was screened for PPO-inhibitor resistance against a rate of fomesafen that completely controlled a susceptible standard. Seeds from each accession were germinated in 50-well plastic trays filled with potting mix (Sunshine ${ }^{\circledR}$ Premix No. 1, Sun Gro Horticulture, Bellevue, WA). One week after germination, seedlings were thinned to 1 plant well $^{-}$. Plants were grown under a 16 -h photoperiod and a 35/25 C day/night temperature. Once seedlings reached a 5- to 7-cm height (3- to 4-leaf stage), they were sprayed with fomesafen at $395 \mathrm{~g}$ ai ha ${ }^{-1}$ (Flexstar ${ }^{\circledR} 1.88$ EC, Syngenta, Greensboro, NC) using a research track sprayer equipped with two flat-fan spray nozzles (TeeJet ${ }^{\circledR}$ spray nozzles, Spraying System, Wheaton, IL) calibrated to deliver $187 \mathrm{~L} \mathrm{ha}^{-1}$ of herbicide solution at $269 \mathrm{kPa}$, moving at $1.6 \mathrm{~km} \mathrm{~h}^{-1}$. A nonionic surfactant was included at $0.25 \% \mathrm{v} / \mathrm{v}$. At $21 \mathrm{~d}$ after treatment (DAT), dead or alive counts were recorded for each accession and expressed as percent mortality. The experiment was conducted in two runs in a completely randomized design with 1 replication run $^{-1}\left(50\right.$ plants run $\left.{ }^{-1}\right)$. All maps presented in this paper were made using the ggplot in R software (R Core Team 2017).

\section{TaqMan $^{\circledast}$ qPCR Assay for Detection of the} $\Delta$ G210 and R128G/M Mutations. After percent mortality was recorded, young leaf tissue was harvested from a maximum of 20 plants from each accession that had survivors. In total, 167 accessions were genotypically screened out of the original 227 accessions in the greenhouse screen. Harvested tissue from each plant was placed into separate $1.5-\mathrm{mL}$ microfuge tubes (Thermo Fisher Scientific, Waltham, MA) and subsequently stored at $-80 \mathrm{C}$ until use. Genomic DNA (gDNA) was isolated from each plant sample using a modified CTAB (cetyl trimethylammonium bromide) protocol (Doyle and Doyle 1987). The TaqMan ${ }^{\circledR}$ quantitative polymerase chain reaction (qPCR) allelic discrimination (AD) assay was performed using a CFX 96 real-time detection system (Bio-Rad, Hercules, CA) to detect the presence/absence of the $\Delta \mathrm{G} 210$ codon deletion or the R128G or R128M mutation in the PPX2 gene of collected plants. Fluorescent probes (6-carboxyfluorescein, FAM and VIC labeled) were used to discriminate between the resistant and susceptible alleles 
of the PPX2 gene in the accessions. For each assay, the qPCR reaction mix $(10 \mu \mathrm{l})$ consisted of $2 \mu \mathrm{l}$ of GoTaq ${ }^{\circledR}$ Flexi buffer (Promega, Madison, WI), $1.2 \mu \mathrm{l}$ of $25 \mathrm{mM} \mathrm{MgCl}_{2}$ (Promega), $0.5 \mu \mathrm{l}$ of $10 \mathrm{mM} \mathrm{dNTP}$ mix (Promega), $0.5 \mu \mathrm{l}$ of primer-probe mix (custom TaqMan $^{\circledR}$ SNP genotyping assay, Thermo Fisher Scientific), $0.1 \mu \mathrm{l} \mathrm{GoTaq}{ }^{\circledR}$ Flexi DNA polymerase (Promega), $1 \mu \mathrm{l}$ of gDNA, $4.7 \mu \mathrm{l}$ of molecular grade water. The qPCR conditions were $95 \mathrm{C}$ for $3 \mathrm{~min}, 40$ cycles of $95 \mathrm{C}$ for $15 \mathrm{~s}$, and an annealing at $60 \mathrm{C}$ for $1 \mathrm{~min}$ followed by a plate read on every cycle. All plates included a homozygous susceptible (1986) and a heterozygous resistant plant $(\Delta \mathrm{G} 210)$ as controls. The Bio-Rad CFX software was used to analyze the qPCR allelic discrimination data expressed in relative fluorescence units. The TaqMan primer and probe combinations used for detection of the $\Delta \mathrm{G} 210$ codon were previously reported in Giacomini et al. (2017). Detection of the $\Delta \mathrm{G} 210$ codon deletion was done for each harvested plant to calculate the resistance frequency within an accession. The resistance frequency (\%) was calculated using the following equation:

$$
\begin{aligned}
\text { Frequency }= & {\left[\begin{array}{c}
\left(\begin{array}{c}
\text { no. of plants homozygous for } \Delta \mathrm{G} 210+ \\
\text { no. of plants heterozygous for } \Delta \mathrm{G} 210
\end{array}\right) \\
\text { total number of plants used for AD assay }
\end{array}\right] } \\
& \times \% \text { survival in the greenhouse }
\end{aligned}
$$

In contrast, gDNA was pooled from up to 10 individual plants within an accession to perform a quick assay for detecting the presence/absence of mutations at the $128^{\text {th }}$ amino acid position of PPX2 gene in each accession. Common forward (5'-TCTTCTGTCA CAGCCAATTTCACA-3') and reverse (5'-CAGA GGACTTACTAGCACAGGAAGA-3') primers were designed to amplify the regions flanking the coding region of the $128^{\text {th }}$ amino acid. The probes used for the R128G assay were 5'-AAATAAAAGGTACA TAGCTAG-3' and 5'-AAATAAAGGGTACATAG CTA-3', whereas probes used for the R128M assay were: 5'-ATAAAAGGTACATAGCTAGAG-3' and 5'-ATAAAATGTACATAGCTAG-3'. The nucleotides that are underlined in the probe sequences indicate wild-type (AGG) and mutated (GGG or ATG) alleles at the R128 region of the PPX2 gene.

\section{Results and Discussion}

Sensitivity of Arkansas Palmer Amaranth Accessions to Fomesafen. To characterize the efficacy of PPO inhibitors in Arkansas, the progeny from 227 accessions were sprayed with a labeled rate of fomesafen $\left(395 \mathrm{~g} \mathrm{ha}^{-1}\right)$. Accessions were grouped based on whether mortality was $\geq 90 \%$ or $<90 \%$ at 21 DAT (Figure 1; Table 1). The 90\% mortality threshold was chosen based on a previously conducted field survey of soybean farmers in which they

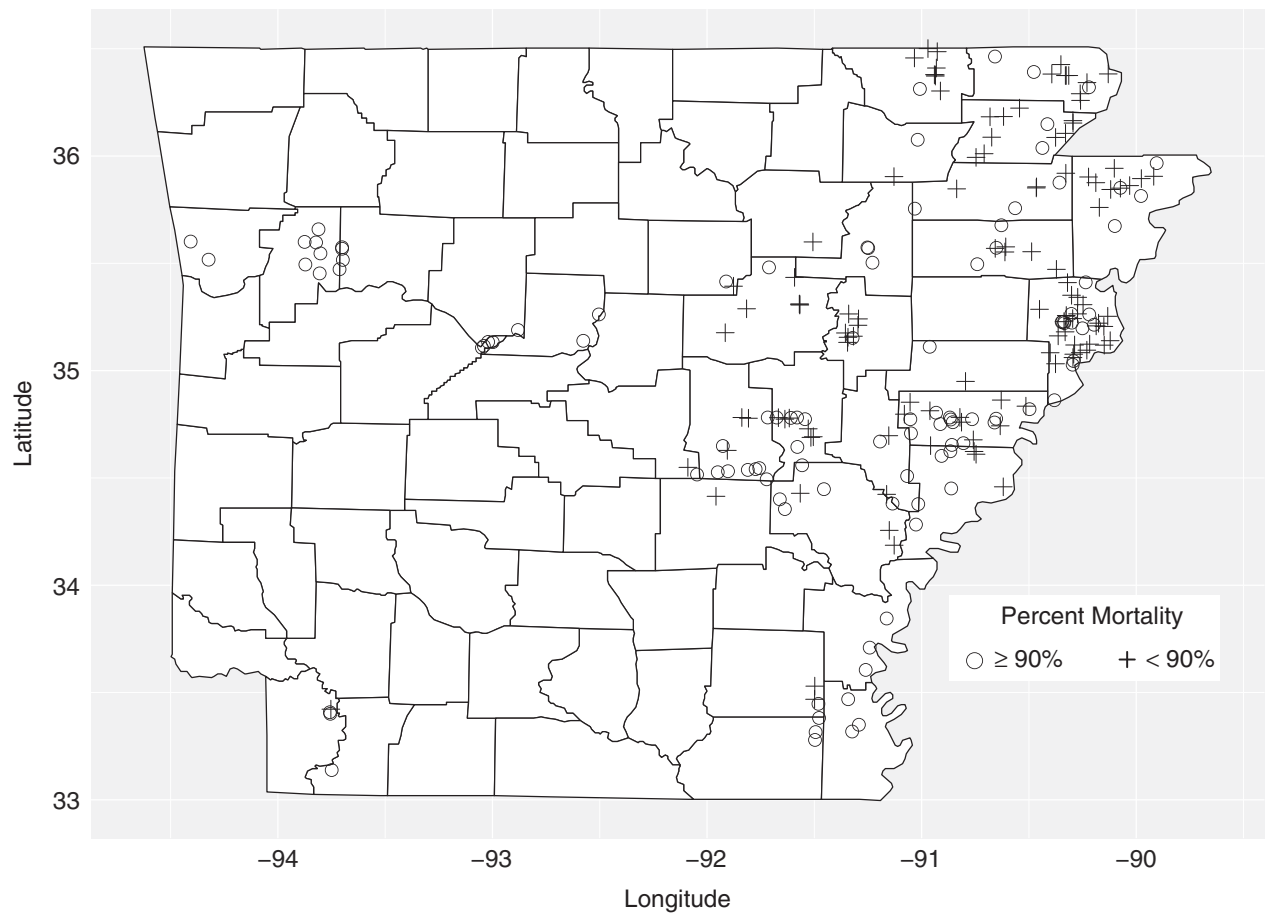

Figure 1. The susceptibility of 227 Palmer amaranth accessions collected from the major row crop-producing areas in Arkansas to fomesafen at $395 \mathrm{~g} \mathrm{ha}^{-1}$. At $21 \mathrm{~d}$ after treatment, dead/alive counts were converted to percent mortality, and accessions were grouped based on whether mortality was $\geq 90 \%$ or $<90 \%$ with fomesafen. 
Table 1. Palmer amaranth accessions treated with $395 \mathrm{~g} \mathrm{ha}^{-1}$ of fomesafen in the greenhouse and screened for PPO-inhibitor resistance. ${ }^{a}$

\begin{tabular}{|c|c|c|c|c|c|c|}
\hline $\begin{array}{l}\text { Palmer } \\
\text { amaranth } \\
\text { accession no. }\end{array}$ & $\begin{array}{l}\% \text { survival } \\
\text { in the } \\
\text { greenhouse }\end{array}$ & $\begin{array}{l}\text { Number of } \\
\text { plants (used for } \\
\text { AD assay) }\end{array}$ & $\begin{array}{l}\text { Number of plants } \\
\text { homozygous for } \\
\Delta \mathrm{G} 210 \text { (allele 1) }\end{array}$ & $\begin{array}{l}\text { Number of plants } \\
\text { heterozygous for } \Delta \mathrm{G} 210 \\
\text { (allele 1/allele 2) }\end{array}$ & $\begin{array}{l}\text { Number of plants } \\
\text { susceptible (negative for } \\
\Delta \text { G210) (allele 2) }\end{array}$ & $\begin{array}{c}\text { Frequency (\%) } \\
\text { of plants with } \\
\Delta \mathrm{G} 210^{\mathrm{b}}\end{array}$ \\
\hline $5 \mathrm{~A}$ & 2 & 1 & - & - & 1 & 0 \\
\hline $7 \mathrm{~A}$ & 2 & 1 & - & - & 1 & 0 \\
\hline $8 \mathrm{~A}$ & 46 & 15 & - & 1 & 14 & 3 \\
\hline $9 \mathrm{~A}$ & 40 & 19 & 1 & 3 & 15 & 8 \\
\hline $10 \mathrm{~A}$ & 6 & 3 & - & - & 3 & 0 \\
\hline $12 \mathrm{~A}$ & 37 & 10 & - & - & 10 & 0 \\
\hline $13 \mathrm{~A}$ & 33 & 17 & - & 15 & 2 & 29 \\
\hline $15 \mathrm{~A}$ & 16 & 12 & - & 7 & 5 & 9 \\
\hline $16 \mathrm{~A}$ & 2 & 1 & - & - & 1 & 0 \\
\hline $18 \mathrm{~A}$ & 41 & 8 & - & 2 & 6 & 10 \\
\hline $20 \mathrm{~A}$ & 38 & 12 & - & - & 12 & 0 \\
\hline $22 \mathrm{~A}$ & 73 & 19 & - & - & 19 & 0 \\
\hline $25 \mathrm{~A}$ & 31 & 14 & - & - & 14 & 0 \\
\hline $26 \mathrm{~A}$ & 6 & 1 & - & - & 1 & 0 \\
\hline $27 \mathrm{~A}$ & 6 & 3 & - & - & 3 & 0 \\
\hline $28 \mathrm{~A}$ & 34 & 19 & - & - & 19 & 0 \\
\hline $29 \mathrm{~A}$ & 51 & 16 & - & 2 & 14 & 6 \\
\hline $30 \mathrm{~A}$ & 13 & 6 & - & - & 6 & 0 \\
\hline $34 \mathrm{~A}$ & 18 & 8 & - & - & 8 & 0 \\
\hline $36 \mathrm{~A}$ & 12 & 5 & - & - & 5 & 0 \\
\hline $37 \mathrm{~A}$ & 6 & 3 & - & - & 3 & 0 \\
\hline $38 \mathrm{~A}$ & 80 & 20 & 1 & 3 & 16 & 16 \\
\hline $39 \mathrm{~A}$ & 56 & 15 & - & - & 15 & 0 \\
\hline $40 \mathrm{~A}$ & 19 & 6 & - & - & 6 & 0 \\
\hline $42 \mathrm{~A}$ & 5 & 3 & - & 3 & - & 5 \\
\hline $43 \mathrm{~A}$ & 40 & 19 & - & - & 19 & 0 \\
\hline $44 \mathrm{~A}$ & 2 & 1 & - & - & 1 & 0 \\
\hline $45 \mathrm{~A}$ & 64 & 14 & 5 & 5 & 4 & 46 \\
\hline $46 \mathrm{~A}$ & 89 & 15 & 9 & 3 & 3 & 71 \\
\hline $47 \mathrm{~A}$ & 6 & 3 & 3 & - & - & 6 \\
\hline $48 \mathrm{~A}$ & 90 & 20 & 5 & 9 & 6 & 63 \\
\hline $49 \mathrm{~A}$ & 63 & 20 & 17 & 3 & - & 63 \\
\hline $50 \mathrm{~A}$ & 78 & 19 & 3 & 4 & 12 & 29 \\
\hline $51 \mathrm{~A}$ & 12 & 6 & - & - & 6 & 0 \\
\hline $52 \mathrm{~A}$ & 62 & 8 & 2 & 5 & 1 & 54 \\
\hline $53 \mathrm{~A}$ & 84 & 20 & 7 & 3 & 10 & 42 \\
\hline $54 \mathrm{~A}$ & 52 & 20 & 8 & 8 & 4 & 42 \\
\hline $55 \mathrm{~A}$ & 6 & 2 & - & - & 2 & 0 \\
\hline $56 \mathrm{~A}$ & 14 & 9 & - & - & 9 & 0 \\
\hline $57 \mathrm{~A}$ & 8 & 6 & - & - & 6 & 0 \\
\hline $58 \mathrm{~A}$ & 16 & 16 & - & 1 & 15 & 1 \\
\hline $59 \mathrm{~A}$ & 36 & 19 & 1 & - & 18 & 2 \\
\hline $60 \mathrm{~A}$ & 11 & 11 & - & - & 11 & 0 \\
\hline $61 \mathrm{~A}$ & 13 & 13 & - & 1 & 12 & 1 \\
\hline $62 \mathrm{~A}$ & 18 & 11 & - & - & 11 & 0 \\
\hline $63 \mathrm{~A}$ & 14 & 7 & - & - & 7 & 0 \\
\hline $64 \mathrm{~A}$ & 6 & 5 & - & - & 5 & 0 \\
\hline $65 \mathrm{~A}$ & 2 & 1 & - & - & 1 & 0 \\
\hline $66 \mathrm{~A}$ & 6 & 6 & - & - & 6 & 0 \\
\hline $67 \mathrm{~A}$ & 10 & 9 & 1 & 1 & 7 & 2 \\
\hline $68 \mathrm{~A}$ & 6 & 5 & 1 & - & 4 & 1 \\
\hline $69 \mathrm{~A}$ & 10 & 10 & - & 2 & 8 & 2 \\
\hline $70 \mathrm{~A}$ & 16 & 5 & - & - & 5 & 0 \\
\hline $71 \mathrm{~A}$ & 14 & 6 & 1 & - & 5 & 2 \\
\hline $72 \mathrm{~A}$ & 30 & 10 & - & - & 10 & 0 \\
\hline $73 \mathrm{~A}$ & 16 & 7 & - & - & 7 & 0 \\
\hline $74 \mathrm{~A}$ & 6 & 4 & - & - & 4 & 0 \\
\hline $75 \mathrm{~A}$ & 8 & 1 & - & - & 1 & 0 \\
\hline
\end{tabular}


Table 1. (Continued)

\begin{tabular}{|c|c|c|c|c|c|c|}
\hline $\begin{array}{l}\text { Palmer } \\
\text { amaranth } \\
\text { accession no. }\end{array}$ & $\begin{array}{l}\text { \% survival } \\
\text { in the } \\
\text { greenhouse }\end{array}$ & $\begin{array}{l}\text { Number of } \\
\text { plants (used for } \\
\text { AD assay) }\end{array}$ & $\begin{array}{l}\text { Number of plants } \\
\text { homozygous for } \\
\Delta \mathrm{G} 210 \text { (allele } 1)\end{array}$ & $\begin{array}{l}\text { Number of plants } \\
\text { heterozygous for } \Delta \mathrm{G} 210 \\
\text { (allele 1/allele } 2)\end{array}$ & $\begin{array}{c}\text { Number of plants } \\
\text { susceptible (negative for } \\
\Delta \text { G210) (allele 2) }\end{array}$ & $\begin{array}{c}\text { Frequency }(\%) \\
\text { of plants with } \\
\Delta \mathrm{G} 210^{\mathrm{b}}\end{array}$ \\
\hline $76 \mathrm{~A}$ & 14 & 1 & - & - & 1 & 0 \\
\hline $77 \mathrm{~A}$ & 24 & 9 & - & - & 9 & 0 \\
\hline $79 A$ & 80 & 18 & 5 & 6 & 7 & 49 \\
\hline $81 \mathrm{~A}$ & 7 & 3 & - & - & 3 & 0 \\
\hline $82 \mathrm{~A}$ & 4 & 1 & - & - & 1 & 0 \\
\hline $83 \mathrm{~A}$ & 73 & 8 & 2 & 3 & 3 & 46 \\
\hline $84 \mathrm{~A}$ & 82 & 16 & 1 & 8 & 7 & 46 \\
\hline $85 \mathrm{~A}$ & 76 & 9 & 1 & 4 & 4 & 42 \\
\hline $87 \mathrm{~A}$ & 68 & 14 & - & 2 & 12 & 9 \\
\hline $89 \mathrm{~A}$ & 26 & 12 & - & - & 12 & 0 \\
\hline $90 \mathrm{~A}$ & 14 & 2 & - & - & 2 & 0 \\
\hline $91 \mathrm{~A}$ & 78 & 18 & 1 & - & 17 & 4 \\
\hline $92 \mathrm{~A}$ & 69 & 19 & 1 & - & 18 & 4 \\
\hline $93 \mathrm{~A}$ & 24 & 20 & - & - & 20 & 0 \\
\hline $97 \mathrm{~A}$ & 5 & 5 & - & 2 & 3 & 2 \\
\hline $101 \mathrm{~A}$ & 48 & 10 & 4 & 5 & 1 & 43 \\
\hline $103 \mathrm{~A}$ & 6 & 3 & 1 & - & 2 & 2 \\
\hline $104 \mathrm{~A}$ & 2 & 1 & - & 1 & - & 2 \\
\hline $105 \mathrm{~A}$ & 6 & 3 & - & 1 & 2 & 2 \\
\hline $106 \mathrm{~A}$ & 33 & 10 & - & - & 10 & 0 \\
\hline $107 \mathrm{~A}$ & 2 & 1 & - & - & 1 & 0 \\
\hline $108 \mathrm{~A}$ & 36 & 9 & - & - & 9 & 0 \\
\hline $109 \mathrm{~A}$ & 45 & 8 & - & 2 & 6 & 11 \\
\hline $110 \mathrm{~A}$ & 83 & 10 & - & 2 & 8 & 17 \\
\hline $111 \mathrm{~A}$ & 18 & 9 & - & 1 & 8 & 2 \\
\hline $112 \mathrm{~A}$ & 10 & 5 & - & - & 5 & 0 \\
\hline $113 \mathrm{~A}$ & 36 & 6 & - & - & 6 & 0 \\
\hline $114 \mathrm{~A}$ & 37 & 9 & - & 3 & 6 & 12 \\
\hline $115 \mathrm{~A}$ & 61 & 9 & - & 1 & 8 & 7 \\
\hline $116 \mathrm{~A}$ & 10 & 5 & - & - & 5 & 0 \\
\hline $118 \mathrm{~A}$ & 96 & 5 & - & 2 & 3 & 38 \\
\hline $119 \mathrm{~A}$ & 77 & 10 & 6 & 1 & 3 & 54 \\
\hline $120 \mathrm{~A}$ & 65 & 5 & - & - & 5 & 0 \\
\hline $121 \mathrm{~A}$ & 61 & 10 & - & 1 & 9 & 6 \\
\hline $122 \mathrm{~A}$ & 34 & 10 & - & - & 10 & 0 \\
\hline $123 \mathrm{~A}$ & 66 & 10 & - & - & 10 & 0 \\
\hline $124 \mathrm{~A}$ & 87 & 10 & - & 2 & 8 & 17 \\
\hline $125 \mathrm{~A}$ & 16 & 10 & - & - & 10 & 0 \\
\hline $126 \mathrm{~A}$ & 48 & 10 & - & - & 10 & 0 \\
\hline $127 \mathrm{~A}$ & 50 & 10 & - & - & 10 & 0 \\
\hline $128 \mathrm{~A}$ & 28 & 10 & - & - & 10 & 0 \\
\hline $129 \mathrm{~A}$ & 19 & 7 & - & - & 7 & 0 \\
\hline $131 \mathrm{~A}$ & 6 & 4 & - & - & 4 & 0 \\
\hline $132 \mathrm{~A}$ & 6 & 3 & - & - & 3 & 0 \\
\hline $139 \mathrm{~A}$ & 12 & 6 & - & - & 6 & 0 \\
\hline $140 \mathrm{~A}$ & 2 & 1 & - & - & 1 & 0 \\
\hline $141 \mathrm{~A}$ & 4 & 2 & - & - & 2 & 0 \\
\hline $145 \mathrm{~A}$ & 6 & 3 & - & - & 3 & 0 \\
\hline $146 \mathrm{~A}$ & 97 & 10 & - & - & 10 & 0 \\
\hline $147 \mathrm{~A}$ & 19 & 8 & - & - & 8 & 0 \\
\hline $148 \mathrm{~A}$ & 81 & 1 & - & - & 1 & 0 \\
\hline $149 \mathrm{~A}$ & 4 & 2 & - & - & 2 & 0 \\
\hline $150 \mathrm{~A}$ & 6 & 3 & - & 2 & 1 & 4 \\
\hline $151 \mathrm{~A}$ & 8 & 4 & - & - & 4 & 0 \\
\hline $152 \mathrm{~A}$ & 38 & 10 & - & - & 10 & 0 \\
\hline $153 \mathrm{~A}$ & 40 & 8 & - & 1 & 7 & 5 \\
\hline $154 \mathrm{~A}$ & 35 & 8 & - & - & 8 & 0 \\
\hline $156 \mathrm{~A}$ & 20 & 10 & - & - & 10 & 0 \\
\hline $157 \mathrm{~A}$ & 2 & 1 & - & 1 & - & 2 \\
\hline
\end{tabular}

Varanasi et al.: Prevalence of PPO resistance • 153 
Table 1. (Continued)

\begin{tabular}{|c|c|c|c|c|c|c|}
\hline $\begin{array}{l}\text { Palmer } \\
\text { amaranth } \\
\text { accession no. }\end{array}$ & $\begin{array}{l}\text { \% survival } \\
\text { in the } \\
\text { greenhouse }\end{array}$ & $\begin{array}{l}\text { Number of } \\
\text { plants (used for } \\
\text { AD assay) }\end{array}$ & $\begin{array}{l}\text { Number of plants } \\
\text { homozygous for } \\
\Delta \mathrm{G} 210 \text { (allele } 1)\end{array}$ & $\begin{array}{l}\text { Number of plants } \\
\text { heterozygous for } \Delta \mathrm{G} 210 \\
\text { (allele 1/allele } 2 \text { ) }\end{array}$ & $\begin{array}{c}\text { Number of plants } \\
\text { susceptible (negative for } \\
\Delta \mathrm{G} 210) \text { (allele 2) }\end{array}$ & $\begin{array}{c}\text { Frequency (\%) } \\
\text { of plants with } \\
\Delta \mathrm{G} 210^{\mathrm{b}}\end{array}$ \\
\hline $159 \mathrm{~A}$ & 35 & 20 & 1 & 8 & 11 & 16 \\
\hline $160 \mathrm{~A}$ & 13 & 13 & 3 & 1 & 9 & 4 \\
\hline $161 \mathrm{~A}$ & 58 & 16 & - & - & 16 & 0 \\
\hline $164 \mathrm{~A}$ & 40 & 16 & 2 & - & 14 & 5 \\
\hline $165 \mathrm{~A}$ & 68 & 19 & 5 & - & 14 & 18 \\
\hline $168 \mathrm{~A}$ & 20 & 7 & - & - & 7 & 0 \\
\hline $169 \mathrm{~A}$ & 40 & 11 & - & 3 & 8 & 11 \\
\hline $178 \mathrm{~A}$ & 1 & 1 & - & - & 1 & 0 \\
\hline $182 \mathrm{~A}$ & 44 & 15 & - & 3 & 12 & 9 \\
\hline $187 \mathrm{~A}$ & 69 & 15 & 1 & - & 14 & 5 \\
\hline $188 \mathrm{~A}$ & 19 & 11 & 1 & 6 & 4 & 12 \\
\hline $189 \mathrm{~A}$ & 20 & 17 & 1 & 1 & 15 & 2 \\
\hline $190 \mathrm{~A}$ & 10 & 5 & - & 1 & 4 & 2 \\
\hline $191 \mathrm{~A}$ & 27 & 14 & 2 & 6 & 6 & 15 \\
\hline $192 \mathrm{~A}$ & 38 & 14 & - & 2 & 12 & 5 \\
\hline $193 \mathrm{~A}$ & 20 & 13 & 2 & - & 11 & 3 \\
\hline $194 \mathrm{~A}$ & 2 & 1 & - & - & 1 & 0 \\
\hline $195 \mathrm{~A}$ & 18 & 11 & - & - & 11 & 0 \\
\hline $196 \mathrm{~A}$ & 28 & 5 & - & - & 5 & 0 \\
\hline $197 \mathrm{~A}$ & 73 & 19 & 5 & 6 & 8 & 42 \\
\hline $198 \mathrm{~A}$ & 28 & 12 & - & 1 & 11 & 2 \\
\hline $216 \mathrm{~A}$ & 22 & 18 & 3 & 12 & 3 & 18 \\
\hline $217 \mathrm{~A}$ & 62 & 19 & - & 11 & 8 & 36 \\
\hline $218 \mathrm{~A}$ & 79 & 18 & 8 & 6 & 4 & 61 \\
\hline $219 \mathrm{~A}$ & 78 & 12 & 4 & 1 & 7 & 33 \\
\hline $220 \mathrm{~A}$ & 90 & 10 & 6 & 3 & 1 & 81 \\
\hline $224 \mathrm{~A}$ & 4 & 2 & 1 & - & 1 & 2 \\
\hline $225 \mathrm{~A}$ & 4 & 2 & - & - & 2 & 0 \\
\hline $227 \mathrm{~A}$ & 2 & 1 & - & - & 1 & 0 \\
\hline $228 \mathrm{~A}$ & 2 & 1 & - & - & 1 & 0 \\
\hline $231 \mathrm{~A}$ & 4 & 1 & - & - & 1 & 0 \\
\hline $233 \mathrm{~A}$ & 3 & 3 & - & - & 3 & 0 \\
\hline $239 \mathrm{~A}$ & 2 & 2 & - & - & 2 & 0 \\
\hline $240 \mathrm{~A}$ & 4 & 1 & - & - & 1 & 0 \\
\hline $241 \mathrm{~A}$ & 4 & 1 & - & - & 1 & 0 \\
\hline
\end{tabular}

${ }^{a}$ Percent survival was calculated out of 100 treated plants. The survivors of the herbicide treatment were subjected to an allelic discrimination (AD) qPCR assay to detect the presence/absence of the $\Delta \mathrm{G} 210$ codon deletion in the PPX2 gene and its corresponding frequency in each accession. The number of homozygous and heterozygous individuals in each accession with respect to the $\Delta \mathrm{G} 210$ deletion conferring PPO-inhibitor resistance is also shown. A dash $(-)$ indicates zero plants were found to contain this genotype.

${ }^{\mathrm{b}}$ Frequency $=\left[\frac{(\text { no. of plants homozygous for } \Delta \mathrm{G} 210+\text { no. of plants heterozygous for } \Delta \mathrm{G} 210)}{\text { total number of plants used for } \mathrm{AD} \text { assay }}\right] \times \%$ survival in the greenhouse 
Table 2. The presence (+) or absence (-) of target-site deletion $(\Delta \mathrm{G} 210)$ and mutations (R128G/M) in the 167 Palmer amaranth accessions from Arkansas. ${ }^{a}$

\begin{tabular}{|c|c|c|c|}
\hline $\begin{array}{l}\text { Palmer amaranth } \\
\text { accession no. }\end{array}$ & $\Delta \mathrm{G} 210$ & $\mathrm{R} 128 \mathrm{G}$ & $\mathrm{R} 128 \mathrm{M}$ \\
\hline $5 \mathrm{~A}$ & - & - & - \\
\hline $7 \mathrm{~A}$ & - & - & - \\
\hline $8 \mathrm{~A}$ & + & + & - \\
\hline $9 \mathrm{~A}$ & + & + & - \\
\hline $10 \mathrm{~A}$ & - & - & - \\
\hline $12 \mathrm{~A}$ & - & - & + \\
\hline $13 \mathrm{~A}$ & + & - & - \\
\hline $15 \mathrm{~A}$ & + & - & - \\
\hline $16 \mathrm{~A}$ & - & - & - \\
\hline $18 \mathrm{~A}$ & + & + & - \\
\hline $20 \mathrm{~A}$ & - & + & - \\
\hline $22 \mathrm{~A}$ & - & + & - \\
\hline $25 \mathrm{~A}$ & - & - & - \\
\hline $26 \mathrm{~A}$ & - & - & - \\
\hline $27 \mathrm{~A}$ & - & - & - \\
\hline $28 \mathrm{~A}$ & - & - & - \\
\hline $29 \mathrm{~A}$ & + & + & - \\
\hline $30 \mathrm{~A}$ & - & - & - \\
\hline $34 \mathrm{~A}$ & - & + & - \\
\hline $36 \mathrm{~A}$ & - & - & - \\
\hline $37 \mathrm{~A}$ & - & - & - \\
\hline $38 \mathrm{~A}$ & + & - & - \\
\hline $39 \mathrm{~A}$ & - & - & - \\
\hline $40 \mathrm{~A}$ & - & - & - \\
\hline $42 \mathrm{~A}$ & + & - & - \\
\hline $43 \mathrm{~A}$ & - & + & - \\
\hline $44 \mathrm{~A}$ & - & - & - \\
\hline $45 \mathrm{~A}$ & + & + & - \\
\hline $46 \mathrm{~A}$ & + & + & - \\
\hline $47 \mathrm{~A}$ & + & - & - \\
\hline $48 \mathrm{~A}$ & + & - & - \\
\hline $49 \mathrm{~A}$ & + & - & - \\
\hline $50 \mathrm{~A}$ & + & - & - \\
\hline $51 \mathrm{~A}$ & - & - & - \\
\hline $52 \mathrm{~A}$ & + & - & - \\
\hline $53 \mathrm{~A}$ & + & - & - \\
\hline $54 \mathrm{~A}$ & + & - & - \\
\hline $55 \mathrm{~A}$ & - & - & - \\
\hline $56 \mathrm{~A}$ & - & + & - \\
\hline $57 \mathrm{~A}$ & - & + & - \\
\hline $58 \mathrm{~A}$ & + & - & - \\
\hline $59 \mathrm{~A}$ & + & + & - \\
\hline $60 \mathrm{~A}$ & - & + & - \\
\hline $61 \mathrm{~A}$ & + & - & - \\
\hline $62 \mathrm{~A}$ & - & + & - \\
\hline $63 \mathrm{~A}$ & - & + & - \\
\hline $64 \mathrm{~A}$ & - & + & - \\
\hline $65 \mathrm{~A}$ & - & - & - \\
\hline $66 \mathrm{~A}$ & - & - & - \\
\hline $67 \mathrm{~A}$ & + & + & - \\
\hline $68 \mathrm{~A}$ & + & - & - \\
\hline $69 \mathrm{~A}$ & + & - & - \\
\hline $70 \mathrm{~A}$ & - & - & - \\
\hline $71 \mathrm{~A}$ & + & + & - \\
\hline $72 \mathrm{~A}$ & - & - & - \\
\hline $73 \mathrm{~A}$ & - & - & - \\
\hline $74 \mathrm{~A}$ & - & - & - \\
\hline $75 \mathrm{~A}$ & - & - & - \\
\hline $76 \mathrm{~A}$ & - & - & - \\
\hline $77 \mathrm{~A}$ & - & - & - \\
\hline $79 A$ & + & + & - \\
\hline $81 \mathrm{~A}$ & - & - & - \\
\hline $82 \mathrm{~A}$ & - & - & - \\
\hline $83 \mathrm{~A}$ & + & - & - \\
\hline $84 \mathrm{~A}$ & + & - & - \\
\hline $85 \mathrm{~A}$ & + & + & - \\
\hline $87 \mathrm{~A}$ & + & - & + \\
\hline $89 \mathrm{~A}$ & - & - & - \\
\hline $90 \mathrm{~A}$ & - & - & - \\
\hline $91 \mathrm{~A}$ & + & - & - \\
\hline $92 \mathrm{~A}$ & + & - & - \\
\hline $93 \mathrm{~A}$ & - & + & - \\
\hline $97 \mathrm{~A}$ & + & - & - \\
\hline $101 \mathrm{~A}$ & + & - & - \\
\hline $103 \mathrm{~A}$ & + & - & - \\
\hline $104 \mathrm{~A}$ & + & - & - \\
\hline $105 \mathrm{~A}$ & + & - & - \\
\hline
\end{tabular}

Table 2. (Continued)

\begin{tabular}{|c|c|c|c|}
\hline $\begin{array}{l}\text { Palmer amaranth } \\
\text { accession no. }\end{array}$ & $\Delta \mathrm{G} 210$ & $\mathrm{R} 128 \mathrm{G}$ & $\mathrm{R} 128 \mathrm{M}$ \\
\hline $106 \mathrm{~A}$ & - & - & - \\
\hline $107 \mathrm{~A}$ & - & - & - \\
\hline $108 \mathrm{~A}$ & - & - & - \\
\hline $109 \mathrm{~A}$ & + & - & - \\
\hline $110 \mathrm{~A}$ & + & + & - \\
\hline $111 \mathrm{~A}$ & + & - & - \\
\hline $112 \mathrm{~A}$ & - & - & - \\
\hline $113 \mathrm{~A}$ & - & + & - \\
\hline $114 \mathrm{~A}$ & + & - & - \\
\hline $115 \mathrm{~A}$ & + & - & - \\
\hline $116 \mathrm{~A}$ & - & + & - \\
\hline $118 \mathrm{~A}$ & + & - & - \\
\hline $119 \mathrm{~A}$ & + & - & - \\
\hline $120 \mathrm{~A}$ & - & + & - \\
\hline $121 \mathrm{~A}$ & + & - & - \\
\hline $122 \mathrm{~A}$ & - & + & - \\
\hline $123 \mathrm{~A}$ & - & - & - \\
\hline $124 \mathrm{~A}$ & + & + & - \\
\hline $125 \mathrm{~A}$ & - & + & - \\
\hline $126 \mathrm{~A}$ & - & - & - \\
\hline $127 \mathrm{~A}$ & - & + & - \\
\hline $128 \mathrm{~A}$ & - & + & - \\
\hline $129 \mathrm{~A}$ & - & + & - \\
\hline $131 \mathrm{~A}$ & - & + & - \\
\hline $132 \mathrm{~A}$ & - & - & - \\
\hline $123 \mathrm{~A}$ & - & + & - \\
\hline $126 \mathrm{~A}$ & - & + & - \\
\hline $139 \mathrm{~A}$ & - & - & - \\
\hline $140 \mathrm{~A}$ & - & - & - \\
\hline $141 \mathrm{~A}$ & - & - & - \\
\hline $145 \mathrm{~A}$ & - & - & - \\
\hline $146 \mathrm{~A}$ & - & - & - \\
\hline $147 \mathrm{~A}$ & - & + & + \\
\hline $148 \mathrm{~A}$ & - & + & - \\
\hline $149 \mathrm{~A}$ & - & - & - \\
\hline $150 \mathrm{~A}$ & + & - & - \\
\hline $151 \mathrm{~A}$ & - & - & - \\
\hline $152 \mathrm{~A}$ & - & + & - \\
\hline $153 \mathrm{~A}$ & + & + & - \\
\hline $154 \mathrm{~A}$ & - & + & - \\
\hline $156 \mathrm{~A}$ & - & - & - \\
\hline $157 \mathrm{~A}$ & + & - & - \\
\hline $158 \mathrm{~A}$ & + & - & - \\
\hline $159 \mathrm{~A}$ & + & - & - \\
\hline $160 \mathrm{~A}$ & + & - & - \\
\hline $161 \mathrm{~A}$ & - & - & - \\
\hline $162 \mathrm{~A}$ & + & - & - \\
\hline $163 \mathrm{~A}$ & + & - & - \\
\hline $164 \mathrm{~A}$ & + & + & - \\
\hline $165 \mathrm{~A}$ & + & - & - \\
\hline $168 \mathrm{~A}$ & - & - & - \\
\hline $169 \mathrm{~A}$ & + & + & - \\
\hline $178 \mathrm{~A}$ & - & - & - \\
\hline $182 \mathrm{~A}$ & + & + & - \\
\hline $183 \mathrm{~A}$ & - & - & - \\
\hline $184 \mathrm{~A}$ & - & - & - \\
\hline $185 \mathrm{~A}$ & + & + & - \\
\hline $186 \mathrm{~A}$ & + & - & - \\
\hline $187 \mathrm{~A}$ & + & - & - \\
\hline $188 \mathrm{~A}$ & + & - & - \\
\hline $189 \mathrm{~A}$ & + & - & - \\
\hline $190 \mathrm{~A}$ & + & - & - \\
\hline $191 \mathrm{~A}$ & + & - & - \\
\hline $192 \mathrm{~A}$ & + & - & - \\
\hline $193 \mathrm{~A}$ & + & + & - \\
\hline $194 \mathrm{~A}$ & - & - & - \\
\hline $195 \mathrm{~A}$ & - & - & - \\
\hline $196 \mathrm{~A}$ & - & - & - \\
\hline $197 \mathrm{~A}$ & + & + & - \\
\hline $198 \mathrm{~A}$ & + & - & - \\
\hline $199 \mathrm{~A}$ & - & - & - \\
\hline $201 \mathrm{~A}$ & + & - & - \\
\hline $202 \mathrm{~A}$ & + & - & - \\
\hline $211 \mathrm{~A}$ & + & - & - \\
\hline $212 \mathrm{~A}$ & + & - & - \\
\hline $213 \mathrm{~A}$ & + & - & - \\
\hline $214 \mathrm{~A}$ & + & - & - \\
\hline $215 \mathrm{~A}$ & + & - & - \\
\hline $216 \mathrm{~A}$ & + & - & - \\
\hline $217 \mathrm{~A}$ & + & - & - \\
\hline
\end{tabular}

Varanasi et al.: Prevalence of PPO resistance • 155 
Table 2. (Continued)

\begin{tabular}{lccc}
\hline $\begin{array}{l}\text { Palmer amaranth } \\
\text { accession no. }\end{array}$ & $\Delta$ G210 & R128G & R128M \\
\hline $218 \mathrm{~A}$ & + & - & - \\
$219 \mathrm{~A}$ & + & - & - \\
$220 \mathrm{~A}$ & + & - & - \\
$224 \mathrm{~A}$ & + & - & - \\
$225 \mathrm{~A}$ & - & - & - \\
$227 \mathrm{~A}$ & - & - & - \\
$228 \mathrm{~A}$ & - & - & - \\
$231 \mathrm{~A}$ & - & - & - \\
$233 \mathrm{~A}$ & - & - & - \\
$239 \mathrm{~A}$ & - & - & - \\
$240 \mathrm{~A}$ & - & - & - \\
$241 \mathrm{~A}$ & - & & \\
\hline
\end{tabular}

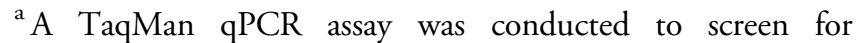
mutation(s) conferring PPO-inhibitor resistance in Palmer amaranth. Out of the 227 total accessions screened in the greenhouse, only 183 accessions had plants alive at $21 \mathrm{~d}$ after fomesafen treatment and a number (16) of accessions had only one or two survivors.

indicated $>90 \%$ mortality as an acceptable level of weed control for POST herbicides (Norsworthy 2003). A known susceptible accession (1986) was included in the screen as a control and, as expected, had $100 \%$ mortality at 21 DAT (unpublished data). Out of 227 accessions, 108 had $\geq 90 \%$ mortality, with 44 accessions having no survivors at 21 DAT (Table 1). In contrast, $52 \%$ of the screened accessions had $<90 \%$ mortality, and these accessions could be found in 21 out of the 29 counties sampled in this study (Figure 1). For example, 24 out of the 39 accessions collected from Crittenden County had $<90 \%$ mortality, indicating growers in this county should assume PPO-inhibiting herbicides will not adequately control Palmer amaranth. In general, accessions located in the northeastern and mideastern (above $35.5^{\circ} \mathrm{N}$ ) parts of Arkansas were poorly controlled ( $<90 \%$ mortality), indicating that gene flow of putative fomesafen resistance alleles is widespread and troublesome in the aforementioned regions.

Identification and Distribution of PPO-Inhibitor Resistance Mechanisms. To confirm resistance and identify the underlying mechanism(s) of resistance to fomesafen in the Arkansas accessions, a TaqMan AD assay was conducted on the surviving plants in the greenhouse screen to detect the presence/absence of the $\Delta \mathrm{G} 210$ and R128G/M mutations in the target-site PPX2 gene (Table 2). These mechanisms are known to confer resistance to PPO-inhibiting herbicides and have been previously reported to exist in a Palmer amaranth accession from Arkansas or in the neighboring state of
Tennessee (Giacomini et al. 2017; Salas et al. 2017). Out of 227 total accessions screened, only 183 accessions had plants alive at 21 DAT; however, a number (16) of accessions had one or two survivors with insufficient tissue for gDNA isolation and therefore were not further analyzed (Table 1). Thus, the TaqMan assay was run on 167 out of the total 227 accessions (Table 2). Several samples were further validated by Sanger DNA sequencing (Genewiz, South Plainfield, NJ), which revealed a high reliability of the TaqMan assay method for screening mutations in the herbicide target-site regions in various accessions (unpublished data).

Distribution of the $\Delta G 210$ Resistance Allele. Of the 167 Palmer amaranth accessions that were genotypically analyzed in this study, only 109 accessions were found to harbor a known PPO inhibitorresistant allelic form of the PPX2 gene (Figure 2; Table 2). PPO inhibitor-resistant Palmer amaranth was found in 18 out of the 29 major agricultureproducing counties in Arkansas, and the $\Delta \mathrm{G} 210$ resistance mechanism was detected in at least one accession from each of those 18 counties (Figure 2). Genotypically, $49 \%$ of the analyzed accessions had at least one plant that harbored an allele of the PPX2 gene encoding a $\Delta G 210$ deletion. The frequency of plants with a $\Delta \mathrm{G} 210$ allele (combined over homozygous and heterozygous plants) ranged from $1 \%$ to $81 \%$, and on average was $20 \%$ (Table 1 ). However, this underestimates the overall frequency of resistant plants, because $27 \%$ of the accessions were found to be segregating for both $\Delta \mathrm{G} 210$ and R128G/M mutations (Table 2). The majority (53\%) of accessions harboring the $\Delta \mathrm{G} 210$ mechanism were located in the northeastern part of the state surrounding the Missouri bootheel (specifically Clay, Greene, Randolph, Lawrence, Craighead, and Mississippi counties) (Figure 2).

Distribution of the R128G and R128M Resistance Alleles. The R128G allele was discovered in $28 \%$ of the 167 accessions screened and found in 12 out of the 29 major agriculture-producing counties in Arkansas (Figure 2; Table 2). Nearly 55\% of the accessions harboring the R128G allele were found in Crittenden and Lee counties near Memphis, TN. The R128M allele, on the other hand, was rare and was found only in one accession each from Prairie (12A), Woodruff (87A), and Crittenden (147A) counties. The large spatial separation of the R128M allele is most likely the result of seed-mediated gene flow or occurred through independent evolutionary 


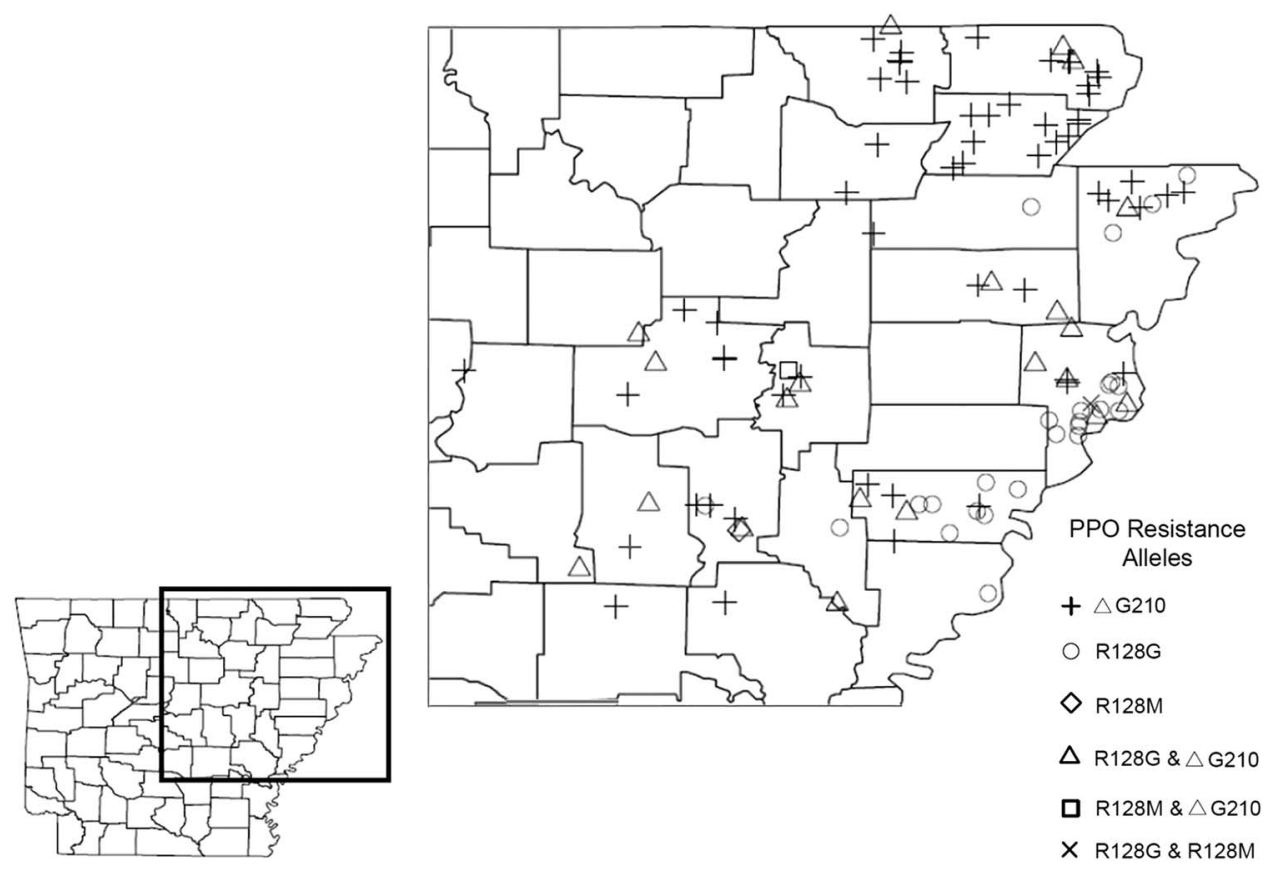

Figure 2. The confirmation and distribution of PPO-inhibitor resistance alleles in Palmer amaranth accessions from Arkansas. A TaqMan qPCR allelic discrimination assay was used to detect the presence or absence of expected target-site resistance mechanisms $(\Delta \mathrm{G} 210$ codon deletion, R128G, and R128M) in the PPX2 gene of Palmer amaranth.

events (Jasieniuk et al. 1996). The frequency of the R128G or R128M allele was not determined, because individuals were pooled within each accession to rapidly screen for the presence/absence of these mutations.

Additionally, 27 out of the 167 accessions genetically screened in this study were not adequately controlled ( $<90 \%$ mortality) with fomesafen and did not contain any known resistant alleles $(\Delta \mathrm{G} 210$ or R128G/M) (Tables 1 and 2). The surviving plants within these accessions exhibited moderate to severe injury; however, by 21 DAT, regrowth was observed from either the apical or the lateral meristems. These accessions are scattered throughout the Arkansas growing region (unpublished data). This may indicate the presence of a novel target-site or non-target site PPO-inhibitor resistance mechanism(s) (such as herbicide metabolism) existing in Arkansas. These 27 accessions need to be investigated further for unknown resistance mechanism(s).

In this study, PPO inhibitor-resistant Palmer amaranth was found in 18 out of the 29 major agriculture-producing counties in Arkansas. This strongly indicates that $\mathrm{PPO}$-inhibitor resistance in Palmer amaranth is widespread in Arkansas, with the most common resistance-conferring alleles being $\Delta$ G210 and R128G (Figure 2; Table 2). These resistance mechanisms have been shown to confer cross-resistance to the PPO-inhibitor chemical families: diphenylethers, $N$-phenylphthalimide, oxadiazole, triazolinone, and pyrimidindione (Rousonelos et al. 2012; Schwartz-Lazaro et al. 2017), indicating growers cannot rely on PPO-inhibiting herbicides to control these biotypes. In fields infested with PPO inhibitor-resistant and glyphosate-resistant Palmer amaranth, growers will need to rotate crops; apply herbicides with different sites of action (e.g., WSSA Groups 5 and 15); use herbicide-resistant trait technologies (LibertyLink ${ }^{\circledR}$, Xtend $^{\circledR}$, or Enlist $\left.{ }^{\circledR}\right)$; and incorporate cultural techniques such as cover crops, tillage, or hand-weeding.

\section{Acknowledgments}

Funding for this research was provided by the Arkansas Soybean Promotion Board.

\section{Literature Cited}

Bond JA, Oliver LR, Stephenson DO IV (2006) Response of Palmer amaranth (Amaranthus palmeri) accessions to glyphosate, fomesafen, and pyrithiobac. Weed Technol 20:885-892

Dayan FE, Daga PR, Duke SO, Lee RM, Tranel PJ, Doerksen RJ (2010) Biochemical and structural consequences of a glycine deletion in the $\alpha-8$ helix of protoporphyringen oxidase. Biochim Biophys Acta 1804:1548-1556

Doyle JJ, Doyle JL (1987) A rapid DNA isolation procedure for small quantities of fresh leaf tissue. Phytochem Bull 19:11-15

Varanasi et al.: Prevalence of PPO resistance • 
Giacomini DA, Umphres-Lopez AM, Nie H, Mueller TC, Steckel LE, Young BG, Tranel PJ (2017) Two new PPX2 mutations associated with resistance to PPO-inhibitor herbicides in Amaranthus palmeri. Pest Manag Sci 73:1559-1563

Heap I (2017) The International Survey of Herbicide Resistant Weeds. http://www.weedscience.org. Accessed: May 15, 2017

Jacobs JM, Jacobs NJ (1993) Porphyrin accumulation and export by isolated barley (Hordeum vulgare) plastids. Plant Physiol 101:1181-1187

Jasieniuk M, Brule-Babel AL, Morrison IN (1996) The evolution and genetics of herbicide resistance in weeds. Weed Sci 44:176-193

Lee RM, Hager AG, Tranel PJ (2008) Prevalence of a novel resistance mechanism to $\mathrm{PPO}$-inhibiting herbicides in waterhemp (Amaranthus tuberculatus). Weed Sci 56:371-375

Matringe MJM, Camadro PL, Scalla R (1989) Protoporphyrinogen oxidase as a molecular target for diphenyl ether herbicides. Biochem J 260:231-235

Norsworthy JK (2003) Use of soybean production surveys to determine weed management needs of South Carolina farmers. Weed Technol 17:195-201

Owen MDK, Zelaya IA (2005) Herbicide-resistant crops and weed resistance to herbicides. Pest Manag Sci 61:301-311

Patzoldt WL, Hager AG, McCormick JS, Tranel PJ (2006) A codon deletion confers resistance to herbicides inhibiting protoporphyrinogen oxidase. Proc Natl Acad Sci USA 103:12329-12334

Prostko EP (2011) Developing a herbicide resistant weed management plan. Page 1538 in Proceedings of the 2011 Beltwide Cotton Conference. Cordova, TN: National Cotton Council of America

R Core Team (2017) R: A Language and Environment for Statistical Computing. Vienna, Austria: R Foundation for Statistical Computing. http://www.R-project.org. Accessed June 12, 2017

Riar DS, Norsworthy JK, Steckel LE, Stephenson DO IV, Eubank TW, Scott RC (2013) Assessment of weed management practices and problem weeds in the Midsouth United States-soybean: a consultant's perspective. Weed Technol 27:612-622

Rousonelos SL, Lee RM, Moreira MS, VanGessel MJ, Tranel PJ (2012) Characterization of a common ragweed (Ambrosia artemisiifolia) population resistant to ALS- and PPO-inhibiting herbicides. Weed Sci 60:335-344

Salas RA, Burgos NR, Rangani G, Singh S, Refatti JP, Piveta L, Tranel PJ, Mauromoustakos A, Scott RC (2017) Frequency of Gly-210 deletion mutation among protoporphyrinogen oxidase inhibitor-resistant Palmer amaranth (Amaranthus palmeri) populations. Weed Sci 65:718-731

Salas RA, Burgos NR, Tranel PJ, Singh S, Glasgow L, Scott RC, Nichols RL (2016) Resistance to PPO-inhibiting herbicide in Palmer amaranth from Arkansas. Pest Manag Sci 72:864-869

Schwartz-Lazaro LM, Norsworthy JK, Scott RC, Barber LT (2017) Resistance of two Arkansas Palmer amaranth populations to multiple herbicide sites of action. Crop Prot 96:158-163

Thinglum KA, Riggins CW, Davis AS, Bradley KW, Al-Khatib K, Tranel PJ (2011) Wide distribution of the waterhemp (Amaranthus tuberculatus) $\triangle \mathrm{G} 210$ PPX2 mutation, which confers resistance to PPO-inhibiting herbicides. Weed Sci 59:22-27

Wychen VL (2016) 2016 Survey of the most common and troublesome weeds in broadleaf crops, fruits \& vegetables in he United States and Canada. Weed Science Society of America National Weed Survey Dataset. http://wssa.net/wp-content/ uploads/2016_Weed_Survey_Final.xlsx. Accessed June 10, 2017

Received July 14, 2017, and approved September 26, 2017.

Associate Editor for this paper: Patrick J. Tranel, University of Illinois. 